\title{
Nuclear Vitamin D Receptor Expression is Associated with Improved Survival in Non-Small Cell Lung Cancer
}

\author{
Malini Srinivasan 1,2 , Anil V. Parwani ${ }^{3}$, Pamela A. Hershberger ${ }^{2,4}$, Diana E. Lenzner ${ }^{5}$, and \\ Joel L. Weissfeld ${ }^{1,2}$ \\ ${ }^{1}$ Department of Epidemiology, University of Pittsburgh Graduate School of Public Health, \\ Pittsburgh, PA 15232, USA \\ 2University of Pittsburgh Cancer Institute, Pittsburgh, PA 15232, USA \\ ${ }^{3}$ Department of Pathology, University of Pittsburgh Medical Center, Pittsburgh, PA 15232, USA \\ ${ }^{4}$ Department of Pharmacology and Chemical Biology, University of Pittsburgh School of Medicine, \\ Pittsburgh, PA 15232, USA \\ ${ }^{5}$ University of Pittsburgh Cancer Institute Biostatistics Facility, Pittsburgh, PA 15232, USA
}

\begin{abstract}
Vitamin D has been shown to have anti-proliferative effects in a wide variety of cancers including lung cancer. The anticancer effects of Vitamin D are mediated primarily by its active metabolite, 1,25-dihydroxyvitamin D (calcitriol), through vitamin D receptor (VDR) signaling. However, thus far there have been no studies evaluating the association between VDR expression and survival outcome in lung cancer. Using immunohistochemical analysis, we evaluated VDR expression, separately in the nucleus and cytoplasm, in lung cancer samples from 73 non-small cell lung carcinoma (NSCLC) patients with no prior therapy, and investigated the association between VDR expression and overall survival (OS). Cox proportional hazard models were used for our primary analyses. There were 44 deaths during a median follow-up of 51 months (range 13-93 months). High nuclear VDR expression was associated with improved OS after adjusting for age, gender, stage, smoking status, and histology (adjusted hazard ratio, 0.36; 95\% confidence interval, 0.17-0.79). There was no association between cytoplasmic VDR expression and OS. Our results suggest that nuclear VDR status may be a prognostic marker in NSCLC. Future large studies to replicate our findings and to assess the impact of VDR gene polymorphisms on VDR expression are required as therapies targeting the vitamin D signaling pathway may be influenced by VDR status in the target lung cancer tissue.
\end{abstract}

\section{Keywords}

Vitamin D receptor; Non-small cell lung cancer; Biomarker; Survival; Prognosis

(C) 2010 Elsevier Ltd. All rights reserved.

Corresponding author: Malini Srinivasan, MD, MPH, UPMC Cancer Pavilion, Fourth floor, Suite 4C, 5150 Centre Avenue, Pittsburgh, PA 15217; Phone: 412-623-3387; Fax: 412-623-3303; srinivasanm3@upmc.edu.

Publisher's Disclaimer: This is a PDF file of an unedited manuscript that has been accepted for publication. As a service to our customers we are providing this early version of the manuscript. The manuscript will undergo copyediting, typesetting, and review of the resulting proof before it is published in its final citable form. Please note that during the production process errors may be discovered which could affect the content, and all legal disclaimers that apply to the journal pertain. 


\section{Introduction}

Vitamin D is a steroid hormone that has a well documented role in calcium homeostasis and bone mineralization. Collective evidence from preclinical and epidemiological studies indicates that vitamin D status is also inversely associated with risk for various cancers and cancer mortality [1,2]. Vitamin D is synthesized in the skin from 7-dehydrocholesterol through solar UV-B exposure or obtained through dietary sources and supplements, is metabolized in the liver to 25-hydroxyvitamin $\mathrm{D}(25(\mathrm{OH}) \mathrm{D})$ and subsequently in the kidney into its active metabolite 1,25-dihydroxyvitamin $\mathrm{D}\left(1,25(\mathrm{OH})_{2} \mathrm{D}\right.$, calcitriol). Vitamin $\mathrm{D}$ and its analogues acting through genomic and non-genomic pathways inhibit cell proliferation, activate apoptotic pathways, inhibit angiogenesis, and exert pro-differentiative effects in a wide variety of cancers. The genomic pathway is mediated by vitamin D receptor (VDR), a 48 to $55 \mathrm{kD}$ protein, and a member of the steroid hormone receptor super family present in various tissues, including lung. VDR is expressed by normal and neoplastic cell types, and is located primarily in the nucleus, although cytoplamic receptors have also been described. VDR is a ligand activated transcription factor. On binding with its ligand $1,25(\mathrm{OH})_{2} \mathrm{D}$, VDR forms a heterodimer with the retinoid-X receptor (RXR), initiates transcription by interacting with a vitamin D response element (VDRE) in the promoter regions of target genes and modulates their expression [3-6].

An inverse association between sunlight and colon cancer mortality was first described in 1980 by Garland \& Garland who hypothesized that vitamin D protects against risk of colon cancer [7]. Subsequent epidemiological studies have shown an association between low circulating 25(OH)D levels, a biomarker of vitamin D exposure, and increased risk for colorectal, breast and prostate cancers [8-10]. An ecologic study showed an independent inverse association between UV-B radiation and vitamin D with reduced mortality at 15 cancer sites [11]. In lung cancer, circulating 25(OH)D levels, along with high vitamin D intake at the time of surgery, was associated with improved survival in early stage non-small cell lung carcinoma (NSCLC) patients [12,13]. Polymorphisms in the VDR gene were also associated with improved survival in early stage NSCLC patients with squamous cell carcinoma in the same cohort [14]. The findings from these epidemiologic studies in lung cancer suggest that the integrity of the vitamin D signaling pathway plays a critical role in influencing outcome in NSCLC. Since the antitumor actions of vitamin D are mediated primarily through VDR [4,5], knowledge of VDR status and its relationship to outcome is important in understanding the natural course of this disease. Additionally, therapeutic efforts targeting this pathway will be influenced by VDR status in the lung cancer tissue. With lung cancer continuing to remain the leading cause of cancer mortality worldwide despite progress in treatment and early detection, there is great interest in developing therapeutic options and preventative approaches for this disease using Vitamin D and its analogues $[15,16]$.

VDR expression has been studied in cancers of the breast, colorectum, kidney, and lung [17-23]. VDR expression was associated with longer survival in human breast cancer [17], colorectal cancer [20], and cholangiocarcinoma [24]. However, there has been no study evaluating the association between VDR expression and survival outcome in lung cancer. Herein, we investigate the association of immunohistochemical expression of VDR with overall survival (OS) in a small cohort of patients with NSCLC. Our hypothesis was that VDR expression would be associated with improved overall survival. We assessed VDR expression in the nucleus and cytoplasm separately in order to evaluate the role of differential VDR expression. 


\section{Materials and Methods}

\subsection{Study Participants and Setting}

The participants of this study belong to one of the cohorts recruited by the Tissue and Blood Bank Core and Biostatistics Core of the Specialized Program of Research Excellence (SPORE) in lung cancer at the University of Pittsburgh Cancer Institute, between July 1997 and January 2006, for translational research projects related to improving the diagnosis and prognosis of patients with NSCLC. It comprises patients with histologically confirmed diagnosis of NSCLC, $\geq 18$ and $\leq 85$ years of age, who underwent surgical resection or surgical staging procedures at the University of Pittsburgh Medical Center (UPMC), and provided written informed consent for collection of blood and tissue samples, and follow-up. There are 90 patients in this cohort. Seven patients in whom only metastatic tissue was available and ten patients who had neoadjuvant therapy were excluded. The current analysis was restricted to the remaining 73 patients who had no prior history of radiation or chemotherapy, whose primary tumor surgical resection tissue was available, and on whom follow-up information was available until April 2009. We assembled risk factor, lung cancer histology, American Joint Committee on Cancer (AJCC) disease stage [25], and follow-up information from outpatient paper charts, inpatient and outpatient electronic medical records, pathology reports, UPMC Cancer Registry, and Social Security Death Index database searches. The date of death was verified with the UPMC Cancer Registry and/or patient's physician. The study was approved by the Institutional Review Board at the University of Pittsburgh.

\subsection{Immunohistochemistry}

Immunohistochemistry was performed with the monoclonal antibody 9A7 (Thermo Scientific) for VDR. Five micrometer thick sections were deparaffinized with 3 rinses of xylene, followed by 2 rinses of absolute alcohol, 1 rinse of $95 \%$ alcohol, 1 rinse of $70 \%$ alcohol, and 2 rinses of deionizer water. Antigen retrieval was performed using DAKO's citrate buffer at pH 6 in a Biocare decloaking chamber. Slides were allowed to cool for 15 minutes and then rinsed several times with deionized water. Endogenous peroxidase was quenched using 3\% hydrogen peroxide for 10 minutes, after which slides were rinsed with deionized water and placed in TBS buffer for 5 minutes. The slides were incubated with 300 $\mu \mathrm{L}$ of primary monoclonal antibody specific for VDR (1:1,000 dilution) for 60 minutes. Slides were then rinsed with TBS buffer, followed by incubation with biotinylated anti-rat $\operatorname{IgG}$ (Vector Laboratories) at 1:300 dilution for 30 minutes. The slides were again rinsed with TBS buffer for 3 minutes, then incubated with DAKO Envision+HRP polymer antirabbit for 30 minutes (DAKO Catalog\# K4003), followed by rinses with TBS buffer for 5 minutes. The DAKO diaminobenzidine (DAB+) chromagen was developed for 10 minutes (DAKO Catalog\# K3468). This was followed by rinsing of the slides in deionized water. All slides were lightly counterstained with Harris hematoxylin for 15 seconds before dehydration, clearing and coverslipping. Appropriate positive and negative controls were included in the staining process; human small intestine was used as positive control, and the negative control consisted of rat IgG2b isotype control serum substituted for primary antibody. Staining was performed on the DAKO Autostainer Plus instrument. All incubations were performed at room temperature.

\subsection{Scoring and Cut-off Selection}

Immunohistochemical expression of VDR was assessed semi-quantitatively for intensity and proportion of positive staining tumor cells without knowledge of patient outcomes. We recorded the proportion (in 5\% increments) of tumor cells expressing VDR, in the nucleus and cytoplasm separately, in each of four integer-scored intensity levels, none (0), weak $(1+)$, moderate (2+), and strong (3+), and a immunohistochemical score for each type of 
VDR expression was calculated. The immunohistochemical score, initially described as histochemical score or 'HSCORE' [26], is a numerical value calculated by finding the sum of the percent staining multiplied by the ordinal value corresponding to each intensity level. Thus, it is a composite score derived by summing the percentage staining multiplied by the weighted intensity of staining, and can have values ranging from 0 to 300 . We used this method as it is an established method of scoring in our laboratory and is also used in clinical practice at our institution for scoring steroid hormone receptors (estrogen receptor, progesterone receptor) in breast cancer. Additionally, although no consistent scoring methods have been used to study steroid hormone receptor expression in lung cancer, two previous studies [27,28] also used a composite scoring method that reflected both the staining intensity and percentage of positive tumor cells.

It was decided a priori to use receiver operating characteristic (ROC) curve analysis [29] for selection of cut-offs for VDR immunohistochemical expression as ROC curves have been recommended as a tool to characterize the performance of immunohistochemical markers and determine cut-off scores for novel biomarkers [30,31]. ROC curve analysis was performed separately for nuclear and cytoplasmic expression scores using mortality (death/ censored) as the end-point, and the score at which the sensitivity and specificity of the outcome are maximized was chosen as the cut-point for dichotomization of the VDR expression score into "high" and "low" expression categories. Low VDR expression was used as the reference category.

\subsection{Statistical Analysis}

The primary end-point of this study was OS, measured as follow-up in months from the date of surgery to the date of death from any cause or until the date of last contact. Patients who were not deceased were censored at the date that they were last known to be alive (date of last contact). Patient demographic and clinical characteristics were compared separately for nuclear and cytoplasmic VDR expression using Pearson's $\chi^{2}$ test (or Fisher's exact test when appropriate) for categorical variables, and Student's t test for continuous variables. Because of the small number of never smokers $(n=3)$, they were combined with the former smokers in the current analysis. The Kaplan-Meier method was used to estimate the OS rates, and log-rank tests were used to compare OS for nuclear and cytoplasmic VDR expression. The 95\% confidence intervals (CI) of the survival rates were computed by applying linear transformation to the survival function $\mathrm{S}(\mathrm{t})$. Cox proportional hazards models were used for our primary analyses to estimate the hazard ratio (HR) and 95\% CI. Factors influencing survival in NSCLC including histology, disease stage, smoking status, age, and sex [32,33] were controlled for in all the multivariate models regardless of their statistical significance in the univariate associations with survival. Age was assessed as a continuous variable. Previous studies $[12,13]$ have shown that vitamin $\mathrm{D}$ is associated with improved survival in early stage (IA-IIB), but not late stage (IIIA-IV) NSCLC. Therefore, we performed subgroup analysis by stage to assess the association of VDR expression with OS in early stage and late stage NSCLC. We also tested for interaction between VDR expression and disease stage by introducing a multiplicative interaction term and assessed the statistical significance using Wald statistic. The Cox proportional hazards assumption was evaluated by examination of the plot of the survival curves $[\log (-\log )$ of the survival distribution function versus $\log$ (follow-up time in months)], and by using the 'assess' statement in SAS version 9.2 which provides a Kolmogorov-type supremum test. There was no violation of the proportional hazards assumption. All statistical tests were two-sided and $\mathrm{p}<0.05$ was considered significant. Statistical analysis was performed using SAS version 9.2 (SAS Institute, Cary, NC). 


\section{Results}

\subsection{Patient Characteristics}

A majority of the 73 NSCLC patients were white (90\%) and current or former smokers (96\%), and two-thirds (66\%) of the patients had early stage (stage IA-IIB) disease. Fifty-six percent of patients were female, and the median age of subjects in this study was 71 years (range 49-85). Adenocarcinoma and squamous cell carcinoma were the predominant histologies, $44 \%$ and $41 \%$ respectively (Table 1 ).

Of the 73 patients, $44(60 \%)$ were deceased and $29(40 \%)$ still alive at the time of last follow-up based on the date of last contact. The median follow-up time was 51 months (range, 13-93 months) for the non-deceased patients, and 33 months (range, 1-104 months) for the entire cohort. The five-year OS rate was 36\% (95\% CI, 23-49\%).

\subsection{VDR Immunohistochemistry}

Based on ROC curve analysis, an immunohistochemical score $>100$ for nuclear VDR expression, and $>20$ for cytoplasmic VDR expression was considered high nuclear and high cytoplasmic expression respectively. The area under the curve (AUC) was 0.56 (95\% CI, $0.42-0.70$ ) for nuclear VDR and 0.52 (95\% CI, 0.38-0.65) for cytoplasmic VDR for the clinical end-point of mortality.

Overall, among the 73 primary NSCLC cases studied, high nuclear and cytoplasmic VDR expression was present in $22(30 \%)$ and $34(47 \%)$ cases respectively. Patient characteristics by nuclear and cytoplasmic VDR expression are summarized in Table 1, and nuclear and cytoplamic VDR expression patterns in various histologies are shown in Figure 1. A higher proportion of men than women had high nuclear expression (11/32 versus 11/41), whereas a higher proportion of women than men had high cytoplasmic VDR expression (21/41 versus 13/32), although these findings were not statistically significant (Table 1). Patient status (death/alive) at last follow-up was significantly associated with nuclear VDR expression with high nuclear VDR expression detected in 59\% of non-deceased and $41 \%$ of deceased patients $(\mathrm{P}=0.026)$. The characteristics of VDR expression (intensity of staining and percentage of stained cells) stratified by patient status at last follow-up are presented in Table 2. Although not statistically significant, for nuclear VDR expression, there was suggestion of a trend toward increasing intensity of staining among non-deceased patients, and decreasing intensity and percentage staining among deceased patients.

\subsection{VDR Expression and Overall Survival}

Patients with high nuclear VDR expression had better OS compared to those with low VDR expression ( $\mathrm{P}=0.077$, log-rank test), with 5-year OS rates of 59\% (95\% CI, 39-80\%) and $27 \%$ (95\% CI, $12-41 \%$ ) respectively (Figure $2 \mathrm{~A})$. In the univariate analysis of Cox proportional hazards model, high nuclear VDR expression was associated with better OS (HR, 0.52; 95\% CI, 0.25-1.09) although this did not reach statistical significance ( $\mathrm{P}=0.085)$. In the multivariate analysis (controlling for age, sex, smoking status, disease stage, histology), there was a statistically significant association between high nuclear VDR expression and overall survival with an adjusted HR of 0.36 (95\% CI, 0.17-0.79; $\mathrm{P}=0.011$; Table 3). This association remained significant even after tumor grade was added as a covariate to the multivariate model (HR, $0.38 ; 95 \% \mathrm{CI}, 0.17-0.82 ; \mathrm{P}=0.015)$.

High cytoplasmic VDR expression was not associated with overall survival in the univariate and multivariate Cox proportional hazard models (Table 3). The five-year OS rate for patients with high cytoplasmic expression was 33\% (95\% CI, 10-55\%), and 36\% (95\% CI, 20-52\%) for those with low cytoplasmic VDR expression (Figure 2B). 
In subgroup analysis by stage, the associations between nuclear VDR expression and OS were statistically similar in early and late stage NSCLC (adjusted HR, 0.34; 95\% CI, 0.11-1.09 versus adjusted HR, 0.39; 95\% CI, 0.11-1.34 respectively; $\mathrm{P}$ for interaction=0.9). The 5-year OS rates were 64\% (95\% CI, 39-89\%) for high nuclear VDR expression and $32 \%$ (95\% CI, 12-52\%) for low nuclear VDR expression in early stage, and 50\% (95\% CI, $15-85 \%)$ and $14 \%$ (95\% CI, 0-31\%) respectively for late stage. Cytoplasmic VDR expression was not associated with OS in early and late-stage NSCLC (Table 3). The 5-year OS rates were $31 \%$ (95\% CI, 2-60\%) for high cytoplasmic expression and 46\% (95\% CI, 23-69\%) for low cytoplasmic expression in early stage. For late stage, the corresponding 5year OS rates were $33 \%$ (95\% CI, 3-64\%) and 21\% (95\% CI, 0.02-42\%) respectively.

\section{Discussion}

We evaluated the association of VDR expression with OS in a small cohort of patients with NSCLC who had received no prior therapy. We found that high nuclear VDR expression was independently associated with better OS in the overall study population. No such association was observed for cytoplasmic VDR expression.

Our results support our hypothesis that VDR expression is associated with improved survival in NSCLC. The role of VDR signaling in mediating improved survival and preventing metastatic growth has been shown in various malignancies in preclinical studies [34-36] and lend support to our findings. In vivo studies have demonstrated that in a defined animal model, acting through VDR, $1,25(\mathrm{OH})_{2} \mathrm{D}$ inhibits metastatic growth of lung carcinoma cell lines [34], while in vitro studies in breast cancer have shown that ablation of VDR shortens survival in MMTV-neu mice [36]. Additionally, a pilot study of human carcinomas (including lung) that measured VDR concentration using an immunoradiometric assay showed that an alteration in VDR number occurs when a cell undergoes malignant transformation and suggested a role for VDR measurement as a marker of prognosis [37].

There have been few studies of VDR expression in lung cancer [22,23]. Although these studies performed immunohistochemistry using the same monoclonal antibody to VDR as our study, they each used a different approach to quantify the immunohistochemical expression of VDR. Kaiser et al [22], detected only nuclear VDR expression in their 37 NSCLC samples, and described the percentages of cells displaying nuclear receptor expression across various histologies. In our study, we detected both nuclear and cytoplasmic VDR expression among the various histological subtypes of NSCLC. Menezes et al [23] performed a descriptive study of VDR immunohistochemical expression across the lung carcinogenesis spectrum in 35 patients with lung cancer and 78 premalignant bronchoscopic biopsy specimens. They assessed the intensity of staining and percentage of positive staining separately for nuclear and cytoplasmic VDR expression, and found that VDR expression was similar among squamous cell carcinoma and adenocarcinoma histologies with more nuclear than cytoplasmic expression, and noted that cytoplasmic VDR was generally lacking in their tumor samples compared to non-tumor samples. We found high nuclear VDR expression among adenocarcinomas and squamous cell carcinomas. A higher proportion of adenocarcinomas in our study also retained high cytoplasmic expression compared to other histologies. Both Menezes et al and Kaiser et al did not evaluate the relation of VDR expression with outcome. Therefore, we were unable to perform a direct comparison of our other study findings.

Our findings suggest that both VDR expression characteristics, intensity of staining and percentage of stained cells, may influence the mortality end-point for nuclear (but not cytoplasmic) VDR expression. 
Upon ligand binding, VDR transits from the cytoplasm to the nucleus, where it regulates gene expression. In colon cancer cells, it has been shown that a cytosolic pool of VDR can also rapidly activate an intracellular signaling cascade (in response to $1,25(\mathrm{OH})_{2} \mathrm{D}$ treatment) that ultimately enhances transcriptional regulation by nuclear VDR [38]. To evaluate the potential importance of this non-genomic signaling pathway, we separately quantified VDR expression in the nucleus and cytoplasm of tumor cells. We observed high cytoplasmic VDR expression in women, and subjects with adenocarcinoma histology. The biological mechanism for this differential VDR expression among certain individual characteristics is unclear. In a recent study of 619 patients with colorectal cancer, cytoplasmic VDR overexpression was independently associated with K-ras and PI3KCA mutations [18]. Additionally, studies conducted in NSCLC cell lines in our laboratory (unpublished data, Hershberger PA) reveal that cell lines with K-ras mutations tend to have lower nuclear VDR expression compared to cell lines without K-ras mutations. K-ras mutations occur predominantly in lung adenocarcinomas, and most of these mutations are smoking-related G-T transversions [39]. Since the majority of patients in our study were current or former smokers, it is possible that they may harbor K-ras mutations that may be responsible for the higher cytoplasmic (and lower nuclear VDR) expression among these patients.

The differences in VDR expression between males and females in our study cannot simply be attributed to differences in estrogen exposure for two reasons. First, the mean age of patients analyzed in our study was 69 . Thus, a majority of the females we analyzed were likely to be post-menopausal. Secondly, recent studies have shown that a majority $(>80 \%)$ of primary lung tumors from both males and females express aromatase, the enzyme that synthesizes estradiol from androgens [40]. Because lung tumors in both males and females have the capacity to locally synthesize and respond to estradiol, any effect of estrogen on VDR expression could potentially occur in both sexes.

VDR activity may also be affected by other members of the Vitamin D signaling pathway [3]. Additionally, polymorphisms in the VDR gene may influence VDR expression in lung cancer tissue and need to be investigated in future studies.

Zhou et al and Heist et al studied the effect of Vitamin D status in early and advanced stage NSCLC respectively [12-14,41]. In a study of 456 patients with early stage NSCLC, Zhou et al found that improved survival was associated with seasonality (surgery during summer) and high recent vitamin D intake [12]. In the same cohort of early stage patients, circulating $25(\mathrm{OH}) \mathrm{D}$ levels were also associated with improved survival, and $\mathrm{Cdx}-2$ polymorphisms in the VDR gene were associated with improved survival in patients with squamous cell carcinoma [13,14]. However, there was no association of survival with circulating $25(\mathrm{OH}) \mathrm{D}$ levels in late stage NSCLC [41]. In our early stage patients, high nuclear (but not cytoplasmic) VDR expression was associated with better survival, and a similar trend was seen in our late stage patients although it was statistically non-significant. Larger studies are warranted for definitive conclusions, and if our findings are confirmed, indicate that vitamin D based therapies may also be beneficial in late stage NSCLC patients with high nuclear VDR expression. In fact, the protective effects of a sufficient vitamin D status on various diseases, including cancer, has already led to recommendations for clinical practice [42].

Our study has many limitations. Our conclusions are limited by the small sample size. However, despite this limitation, nuclear VDR expression was independently associated with improved overall survival in NSCLC after controlling for some of the important determinants of survival suggesting that the effect size was large enough to be significant. A major limitation is the absence of serum $25(\mathrm{OH}) \mathrm{D}$ levels in our NSCLC patients. Hence, we were unable to evaluate the joint effects of VDR expression and serum $25(\mathrm{OH}) \mathrm{D}$ levels. Our 
smoking status information was based only on data collected at the time of recruitment, and is subject to recall bias. For the survival analysis, we assessed OS only as we were unable to distinguish deaths due to lung cancer from deaths due to other causes. Additionally, other factors that influence survival in lung cancer such as performance status, adjuvant therapy after surgical resection were not assessed. Other unadjusted factors in our study include solar UV-B exposure, season of surgery, dietary intake, and body mass index, all of which influence Vitamin D levels. A majority of our study population was Caucasian. This may influence vitamin D status as dark-skinned individuals require more exposure to UV-B radiation than light-skinned individuals for producing an equivalent amount of vitamin $\mathrm{D}$ [1]. Another limitation is generalizability of findings to other populations as our study is based on a convenient sample of patients who were recruited based on certain eligibility criteria and underwent surgical resection of their primary lung cancer at a tertiary care hospital (UPMC). Despite the limitations, there is biological plausibility to support our study results, and even the upper limit of our estimate indicates a $21 \%$ improvement in overall survival for patients with high nuclear VDR expression after adjusting for some other important predictors of survival in NSCLC. These findings suggest a role for VDR as a biological marker of prognosis in NSCLC. The patients in our study received no prior neoadjuvant chemotherapy or radiation therapy, thereby eliminating any bias associated with the predictive value of VDR on outcome that may be related to prior therapy. There was minimal missing data in our study.

\section{Conclusion}

In conclusion, the findings of our study, the first to evaluate the association of VDR expression with survival in NSCLC, although limited by sample size, show an independent association of nuclear VDR expression with better overall survival and suggest that analogous to estrogen receptor status in breast cancer, VDR may be a prognostic marker in NSCLC. Future large studies with more diverse racial groups to confirm the validity of our results, and to assess the impact of interaction of other factors in the vitamin D signaling pathway with VDR expression are warranted.

\section{Acknowledgments}

We thank Dr. Jane Cauley and Dr. Douglas Potter for insightful discussions; Ms Marie Acquafondata and Ms Marianne Notaro for technical assistance.

Funding Source: This work was supported in part by the University of Pittsburgh Specialized Program of Research Excellence (SPORE) in Lung Cancer NCI P50 CA90440, and NIH RO1 CA132844 to P.A.H.

\section{References}

[1]. Giovannucci E. The epidemiology of vitamin D and cancer incidence and mortality: a review (United States). Cancer Causes Control 2005;16:83-95. [PubMed: 15868450]

[2]. Pilz S, Tomaschitz A, Obermayer-Pietsch B, Dobnig H, Pieber TR. Epidemiology of vitamin D insufficiency and cancer mortality. Anticancer Res 2009;29:3699-3704. [PubMed: 19667167]

[3]. Deeb KK, Trump DL, Johnson CS. Vitamin D signalling pathways in cancer: potential for anticancer therapeutics. Nat Rev Cancer 2007;7:684-700. [PubMed: 17721433]

[4]. Beer TM, Myrthue A. Calcitriol in cancer treatment: from the lab to the clinic. Mol Cancer Ther 2004;3:373-81. [PubMed: 15026558]

[5]. Johnson CS, Hershberger PA, Bernardi RJ, Mcguire TF, Trump DL. Vitamin D receptor: a potential target for intervention. Urology 2002;60:123-30. [PubMed: 12231068]

[6]. Barsony J, Renyi I, McKoy W. Subcellular distribution of normal and mutant vitamin D receptors in living cells. Studies with a novel fluorescent ligand. J Biol Chem 1997;272:5774-82.

[PubMed: 9038191] 
[7]. Garland CF, Garland FC. Do sunlight and vitamin D reduce the likelihood of colon cancer? Int J Epidemiol 1980;9:227-231. [PubMed: 7440046]

[8]. Garland CF, Comstock GW, Garland FC, Helsing KJ, Shaw EK, Gorham ED. Serum 25hydroxyvitamin D and colon cancer:eight year prospective study. Lancet 1989;2:1176-78. [PubMed: 2572900]

[9]. Bertone-Johnson ER, Chen WY, Holick MF, Hollis BW, Colditz GA, Willett WC, et al. Plasma 25-hydroxyvitamin D and 1,25-dihydroxyvitamin D and risk of breast cancer. Cancer Epidemiol Biomarkers Prev 2005;14:1991-7. [PubMed: 16103450]

[10]. Ahonen MH, Tenkanen L, Teppo L, Hakama M, Tuohimaa P. Prostate cancer risk and prediagnostic serum 25-hydroxyvitamin D levels (Finland). Cancer Causes Control 2000;11:847852. [PubMed: 11075874]

[11]. Grant WB, Garland CF. The association of solar ultraviolet B (UVB) with reducing risk of cancer: multifactorial ecologic analysis of geographic variation in age-adjusted cancer mortality rates. Anticancer Res 2006;26:2687-2699. [PubMed: 16886679]

[12]. Zhou W, Suk R, Liu G, Park S, Neuberg DS, Wain JC, et al. Vitamin D is associated with improved survival in early-stage non-small cell lung cancer patients. Cancer Epidemiol Biomarkers Prev 2005;14:2303-2309. [PubMed: 16214909]

[13]. Zhou W, Heist RS, Liu G, Asomaning K, Neuberg DS, Hollis BW, et al. Circulating 25hydroxyvitamin D levels predict survival in early-stage non-small-cell lung cancer patients. J Clin Oncol 2007;25:479-485. [PubMed: 17290055]

[14]. Zhou W, Heist RS, Liu G, Neuberg DS, Asomaning K, Su L, et al. Polymorphisms of vitamin D receptor and survival in early-stage non-small cell lung cancer patients. Cancer Epidemiol Biomarkers Prev 2006;15:2239-2245. [PubMed: 17119052]

[15]. Parkin DM, Bray F, Ferlay J, Pisani P. Global cancer statistics, 2002. CA Cancer J Clin 2005;55:74-108. [PubMed: 15761078]

[16]. Trump DL, Deeb KK, Johnson CS. Vitamin D: considerations in the continued development as an agent for cancer prevention and therapy. Cancer J 2010;16:1-9. [PubMed: 20164683]

[17]. Berger U, McClelland RA, Wilson P, Greene GL, Haussler MR, Pike JW, et al. Immunocytochemical determination of estrogen receptor, progesterone receptor, and 1,25dihydroxyvitamin D3 receptor in breast cancer and relationship to prognosis. Cancer Res 1991;51:239-244. [PubMed: 1846309]

[18]. Kure S, Nosho K, Baba Y, Irahara N, Shima K, $\mathrm{Ng} \mathrm{K}$, et al. Vitamin D receptor expression is associated with PIK3CA and KRAS mutations in colorectal cancer. Cancer Epidemiol Biomarkers Prev 2009;18:2765-2772. [PubMed: 19789368]

[19]. Thomas MG, Sylvester PA, Newcomb P, Longman RJ. Vitamin D receptor expression in colorectal cancer. J Clin Pathol 1999;52:181-183. [PubMed: 10450176]

[20]. Evans SRT, Nolla J, hanfelt J, Shabahang M, Nauta RJ, Shchepotin IB. Vitamin D Receptor Expression as a Predictive Marker of Biological Behavior in Human Colorectal Cancer. Clinical Cancer Research 1998;4:1591-1595. [PubMed: 9676831]

[21]. Blomberg JM, Andersen CB, Nielsen JE, Bagi P, Jorgensen A, Juul A, et al. Expression of the vitamin D receptor, 25-hydroxylases, 1alpha-hydroxylase and 24-hydroxylase in the human kidney and renal clear cell cancer. J Steroid Biochem Mol Biol. April 1;2010 Epub ahead of print.

[22]. Kaiser U, Schilli M, Wegmann B, Barth P, Wedel S, Hofmann J, et al. Expression of vitamin D receptor in lung cancer. J Cancer Res Clin Oncol 1996;122:356-359. [PubMed: 8642046]

[23]. Menezes RJ, Cheney RT, Husain A, Tretiakova M, Loewen G, Johnson CS, et al. Vitamin D receptor expression in normal, premalignant, and malignant human lung tissue. Cancer Epidemiol Biomarkers Prev 2008;17:1104-1110. [PubMed: 18483332]

[24]. Seubwai W, Wongkham C, Puapairoj A, Khuntikeo N, Wongkham S. Overexpression of vitamin $\mathrm{D}$ receptor indicates a good prognosis for cholangiocarcinoma: implications for therapeutics. Cancer 2007;109:2497-2505. [PubMed: 17487855]

[25]. Greene, FL.; Page, DL.; Fleming, ID., et al., editors. American Joint Committee on Cancer Staging Manual. sixth ed.. Springer; Philadelphia: 2002. 
[26]. McCarty KS Jr. Miller LS, Cox EB, Konrath J, McCarty KS Sr. Estrogen receptor analyses. Correlation of biochemical and immunohistochemical methods using monoclonal antireceptor antibodies. Arch Pathol Lab Med 1985;109:716-721. [PubMed: 3893381]

[27]. Kawai H, Ishii A, Washiya K, Konno T, Kon H, Yamaya C, et al. Estrogen receptor alpha and beta are prognostic factors in non-small cell lung cancer. Clin Cancer Res 2005;11:5084-5089. [PubMed: 16033821]

[28]. Ali G, Donati V, Loggini B, Servadio A, Dell'Omodarme M, Prati MC, et al. Different estrogen receptor beta expression in distinct histologic subtypes of lung adenocarcinoma. Hum Pathol 2008;39:1465-1473. [PubMed: 18620727]

[29]. Hanley JA. Receiver operating characteristic (ROC) methodology: the state of the art. Crit Rev Diagn Imaging 1989;29:307-335. [PubMed: 2667567]

[30]. True L, Feng Z. Immunohistochemical validation of expression microarray results. J Mol Diagn 2005;7:149-151. [PubMed: 15858137]

[31]. Zlobec I, Steele R, Terracciano L, Jass JR, Lugli A. Selecting immunohistochemical cut-off scores for novel biomarkers of progression and survival in colorectal cancer. J Clin Pathol 2007;60:1112-1116. [PubMed: 17182662]

[32]. Molina JR, Yang P, Cassivi SD, Schild SE, Adjei AA. Non-small cell lung cancer: epidemiology, risk factors, treatment, and survivorship. Mayo Clin Proc 2008;83:584-594. [PubMed: 18452692]

[33]. Wheatley-Price P, Blackhall F, Lee SM, Ma C, Ashcroft L, Jitlal M, et al. The influence of sex and histology on outcomes in non-small-cell lung cancer: a pooled analysis of five randomized trials. Ann Oncol 2010;21:2023-2028. Epub 2010 March 23. [PubMed: 20332134]

[34]. Nakagawa K, Kawaura A, Kato S, Takeda E, Okano T. 1 alpha,25-Dihydroxyvitamin D(3) is a preventive factor in the metastasis of lung cancer. Carcinogenesis 2005;26:429-440. [PubMed: 15539405]

[35]. Eisman JA, Barkla DH, Tutton PJ. Suppression of in vivo growth of human cancer solid tumor xenografts by 1,25-dihydroxyvitamin D3. Cancer Res 1987;47:21-25. [PubMed: 3024816]

[36]. Zinser GM, Welsh J. Vitamin D receptor status alters mammary gland morphology and tumorigenesis in MMTV-neu mice. Carcinogenesis 2004;25:2361-2372. [PubMed: 15333467]

[37]. Sandgren M, Danforth L, Plasse TF, DeLuca HF. 1,25-Dihydroxyvitamin $D_{3}$ Receptors in Human Carcinomas: A Pilot Study. Cancer Research 1991;51:2021-2024. [PubMed: 1849039]

[38]. Ordóñez-Morán P, Muñoz A. Nuclear receptors: genomic and non-genomic effects converge. Cell Cycle 2009;8:1675-1680. [PubMed: 19448403]

[39]. Herbst RS, Heymach JV, Lippman SM. Lung cancer. N Engl J Med 2008;359:1367-1380. [PubMed: 18815398]

[40]. Weinberg OK, Marquez-Garban DC, Fishbein MC, Goodglick L, Garban HJ, Dubinett SM, et al. Aromatase inhibitors in human lung cancer therapy. Cancer Res 2005;65:11287-11291. [PubMed: 16357134]

[41]. Heist RS, Zhou W, Wang Z, Liu G, Neuberg D, Su L, et al. Circulating 25-hydroxyvitamin D, VDR polymorphisms, and survival in advanced non-small-cell lung cancer. J Clin Oncol 2008;26:5596-5602. [PubMed: 18936471]

[42]. Souberbielle JC, Body JJ, Lappe JM, Plebani M, Shoenfeld Y, Wang TJ, et al. Vitamin D and musculoskeletal health, cardiovascular disease, autoimmunity and cancer: Recommendations for clinical practice. Autoimmune Rev 2010;9:709-715. Epub 2010 Jul 1. 


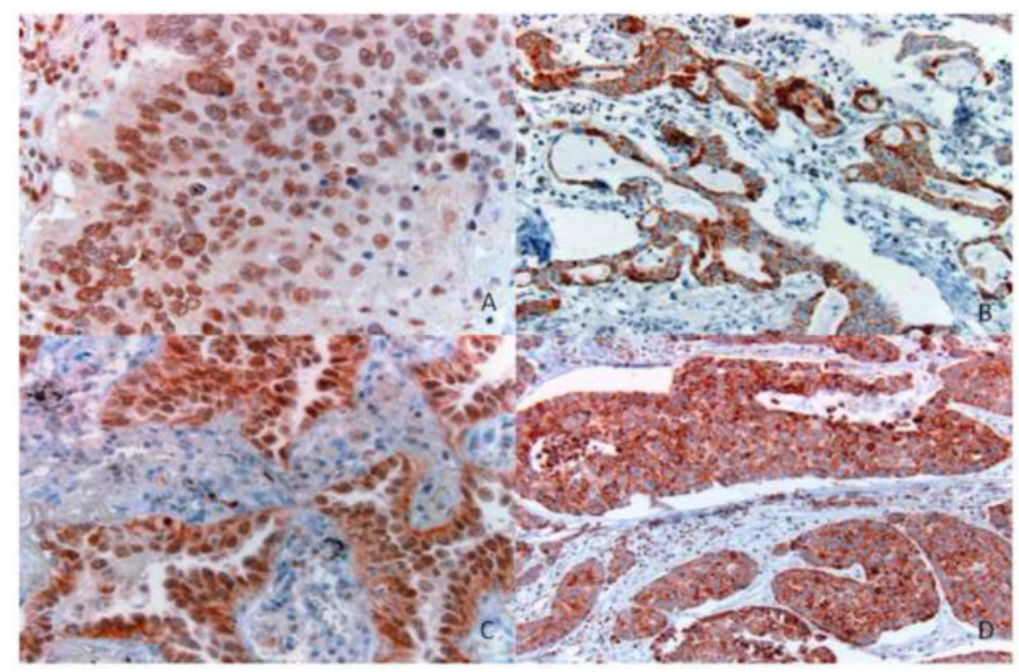

Figure 1.

VDR immunohistochemical expression across various histologies. A. Nuclear VDR expression, squamous cell carcinoma. B. Cytoplasmic VDR expression, adenocarcinoma. C. Nuclear and cytoplasmic VDR expression, adenocarcinoma. D. Cytoplasmic VDR expression, large cell carcinoma. (Original magnification. A, C: 400X; B, D: 200X). VDR: Vitamin D receptor 

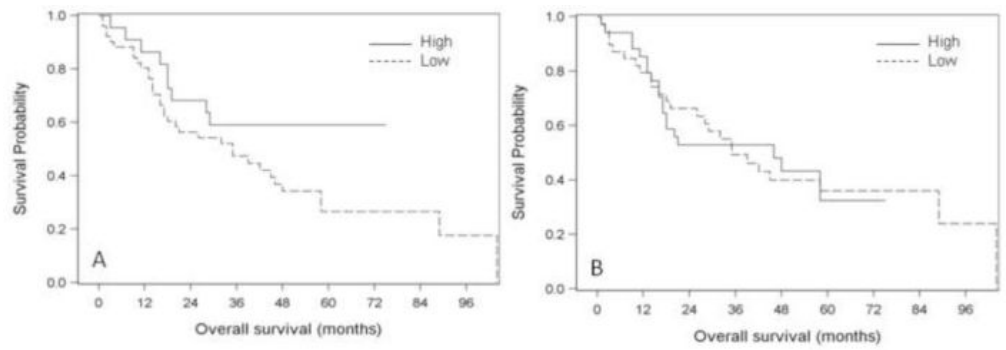

Figure 2.

Kaplan-Meier survival curves for nuclear and cytoplasmic VDR expression. A. Nuclear VDR expression. Patients with high nuclear VDR expression had better probability of OS compared to those with low nuclear VDR expression ( $\mathrm{P}=0.077$, log-rank test). $\mathrm{B}$.

Cytoplasmic VDR expression. No difference in OS between patients with high versus low cytoplasmic VDR expression ( $\mathrm{P}=0.963$, log-rank test). VDR: Vitamin D receptor; OS:

Overall survival 


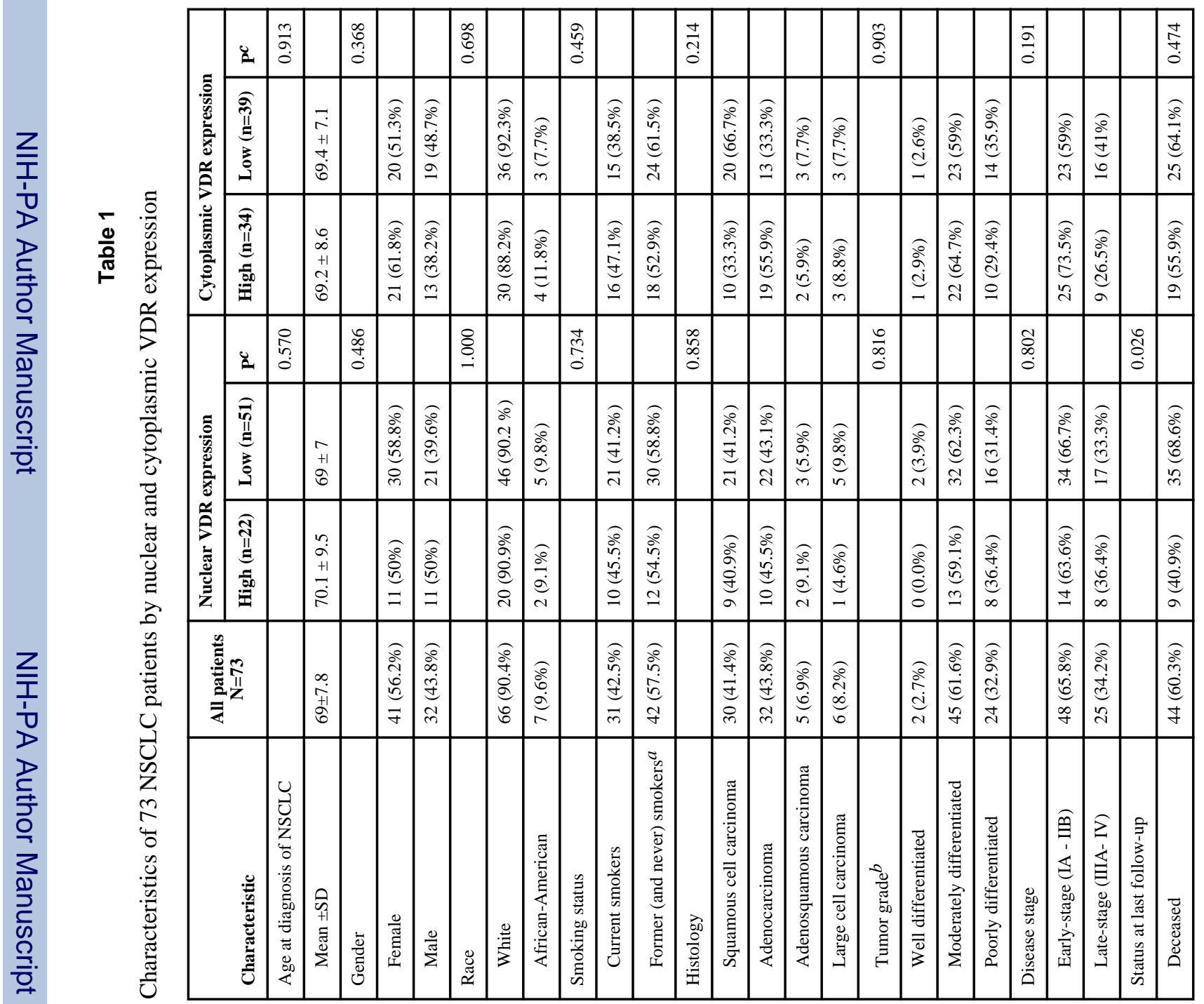




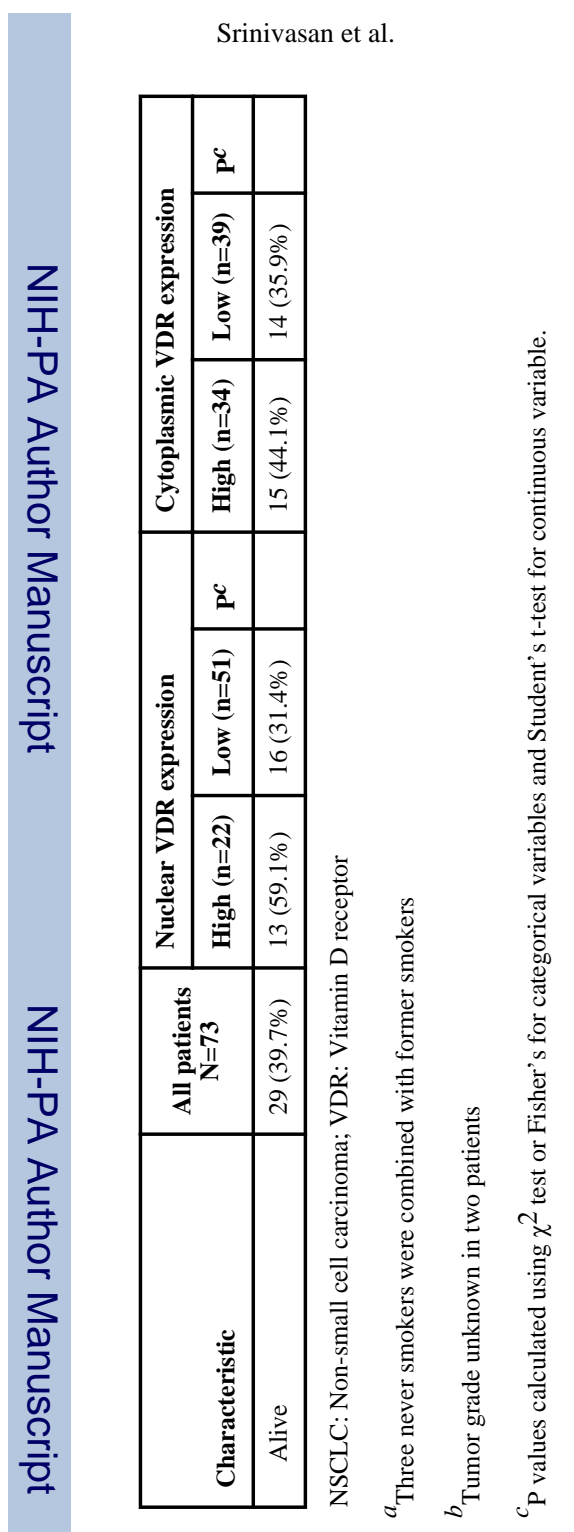

Page 14

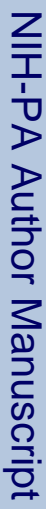

J Steroid Biochem Mol Biol. Author manuscript; available in PMC 2012 January 1. 
